\title{
Laboratory varietal control as a guarantee of successful work of agribusiness in Russia
}

\author{
Anna Lyubimova ${ }^{1,2,{ }^{*}}$ and Dmitry Eremin ${ }^{2}$ \\ ${ }^{1}$ Federal Research Center Tyumen Scientific Center of the Siberian Branch of the Russian Academy \\ of Sciences, 625501 Moskowskiy, Burlaki Street, 2, Tyumen district, Tyumen region, Russia \\ ${ }^{2}$ The State Agrarian University of Northern Zauralye, 625003 Tyumen, Republic Street, 7. Russia
}

\begin{abstract}
A new direction of agribusiness has been formed in the modern agricultural industry. This is expressed in the appearance of highly specialized enterprises working in the field of production of high-quality seeds of agricultural crops. The use of electrophoresis method in varietal identification is a new technology in domestic agribusiness. The purpose of the research was to study the electrophoresis role of prolamins in varietal identification of crops for high-quality seed material. The laboratory of varietal identification of seeds analyzed 47 varieties of oats Russian selection. On the basis of data on the component composition of avenin, varieties with a high level of intersort genetic differences were identified: Megion, Fobos, Local (K-8427), Uspekh, Otrada, Pushkinskij. Groups of samples with identical component composition of prolamins were found. Their genetic formulas of avenin have the following form: $A v n A 2 B 4 C 2, A v n$ $A 4 B 4 C 2, A v n A 2 B 4 C 1$ or $A v n A 2 B 1 C 3$. It is established that the method of electrophoresis of oat prolamins allows effectively distinguish varieties belonging to the same variety and indistinguishable by morphological features. Implementation of a system of regular laboratory control of purity and compliance of original and reproductive oats seeds by electrophoresis of prolamins is necessary to improve the competitiveness of Russian grain production in the world market.
\end{abstract}

\section{Introduction}

For today, agriculture in Russia, despite the imposed sanctions against the country, is actively developing and trying to reach the same level with world leaders in the field of food production. Modern agribusiness represents the various spheres of production activities associated with agrarian sector, starting from the cultivation of products and ending with the processing of it. At all stages, technologies are used to reduce production costs or increase crop yields. However, the main factor on which the effect of the introduction of any technology depends is the seed. Crop yields and the cost of production depend on its quality.

Historically, the agricultural enterprises themselves harvest the seeds of grain crops. And in each economy at least 2-3 grades of each culture are cultivated. This approach can be easily explained. In Russia, and especially in Western Siberia, climatic conditions are very difficult

\footnotetext{
* Corresponding author: ostapenkoav88@yandex.ru
} 
and it is extremely dangerous to rely on one variety. This can lead to a serious shortfall in the crop, if the selected variety is not resistant to any weather factor. However, the presence of several varieties in the economy leads to the risk of varietal blockage. At the use for sowing, harvesting and refining of grain of the same technique, with insufficient cleaning it from the seeds of the previous grade, there is a high probability of mechanical mixing of varieties. It is difficult to detect it visually, since many modern varieties, referring to the same variety, are difficult to distinguish by morphological features.

Even when one variety is used in the household, there is always a certain probability of loss of varietal qualities, since modern varieties of grain crops are complex populations. They are harder to maintain in the process of seed production, as members of such varieties of biotypes, differing in biological properties, can hardly be different according to the morphological characteristics.

The way out of this situation is the periodic acquisition of seed material in seed farms. Their commodity production is a high-quality seed material which possesses high grade purity and the best sowing qualities. In Russia, this sphere of agribusiness began to develop actively 20-30 years ago. However, they also have certain problems related to varietal control. In Russia, to determine the varietal qualities of seeds, field testing and ground control based on morphological characteristics of the variety are generally accepted. Field testing requires highly qualified personnel capable of visually distinguishing the varietal admixture in crops and calculating the percentage of its occurrence in the main batch of seeds. In the conditions of modern agribusiness, this method is unacceptable, because, despite the actual financial and time costs incurred by the economy, the result may be unsatisfactory. Therefore, varietal control is necessary at all stages of seed production. In this regard, there was a need to introduce new technologies in seed production.

Methods based on advances in genetics and physiologies, including the method of electrophoresis of spare alcohol - soluble proteins of seed-prolamins [1, 2, 3] are increasingly used. The advantages of this method include comparative simplicity, low cost and speed of analysis. The composition of the prolamins spectra are stable and do not depend on the growing conditions of plants $[4,5]$.

The purpose of researches. Study the role of electrophoresis of prolamins in the varietal identification of crops to obtain high-quality seed material.

Spare alcohol-soluble proteins of grain - avenins - successfully applied to the analysis of genetic diversity of oats. Due to the significant polymorphism by molecular weight and the number of components, each variety or biotype is characterized by a specific composition of prolamins components. The components of the avenin electrophoretic spectra are inherited by groups and controlled by three independent loci $A v n A, A v n B, A v n C$ [6].

\section{Materials and Methods}

The material for the study was 47 varieties of oats, which were created in the leading breeding centers of the Russian Federation. Varieties of the four most common species were selected for analysis: mutica - plants with a spreading, versatile panicle, white film grain and awnless s pikelets (Fig.1a); aristata - plants with a spreading, versatile panicle, white film grain and spikelets with awn; aurea - plants with a spreading, versatile panicle, yellow film grain and spikelets without awn; and inermis - spreading panicle, bare grain with white floral scales and spikelets without awns (Fig.1b). Oat plants of different varieties are quite distinct, but the varieties of the same species have similar morphological characteristics and are virtually indistinguishable from each other.

The study material provided by Federal Agency of Scientific Organizations Federal State Budgetary Scientific Institution Federal Research Center The N.I. Vavilov All-Russian Institute of plant genetic resources. 


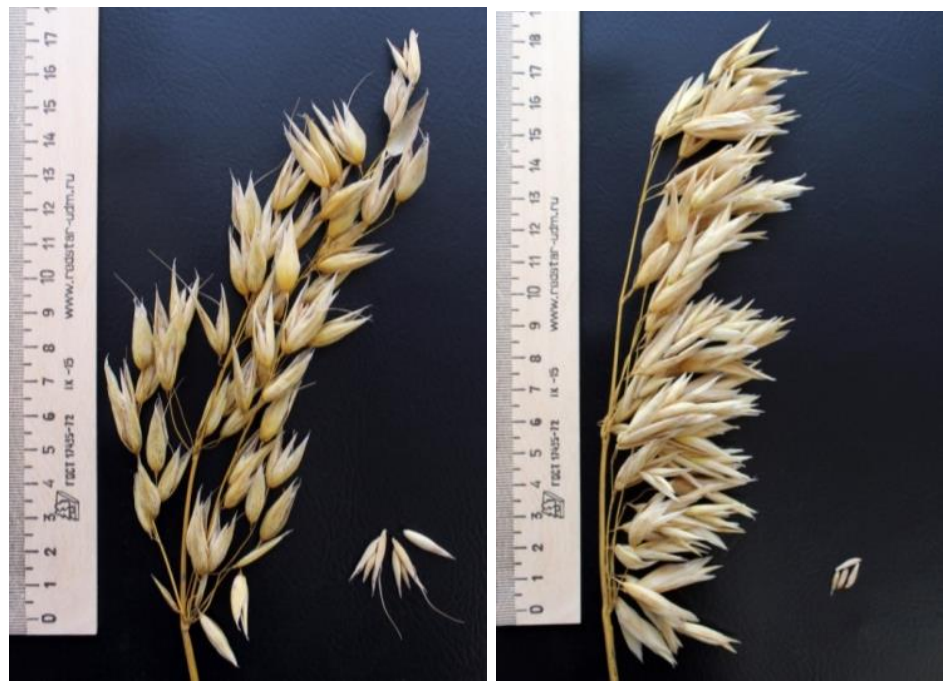

Fig. 1. Appearance of oat plants the most common varieties: a) mutica; b) inermis.

For one-dimensional electrophoresis of spare oats proteins, a standard technique was used [7], with some modifications. For the analysis from each grade by method of random sampling 100 grains were selected. Proteins extracted from the flour of individual grains with the addition of $90 \mathrm{mcl}$ of $70 \%$ ethanol with followed incubation at $40^{\circ} \mathrm{C}$ for 40 minutes. The resulting extract was centrifuged 4 minutes at a speed of 10,000 revolutions per minute. The supernatant was transferred to the new Eppendorf tubes and poured $300 \mathrm{mcl}$ of methylene green dye ( $60 \mathrm{~g}$ of sucrose, $0.1 \mathrm{~g}$ of methylene green, $100 \mathrm{~g}$ of urea and $100 \mathrm{ml}$ of alluminium lactate buffer). The protein extract $(23 \mathrm{mcl})$ was placed in a polyacrylamide gel and separated by electrophoresis. Polyacrylamide gel contained: $13,17 \mathrm{~g}$ of acrylamide, 0,66 $\mathrm{g}$ of $\mathrm{N}, \mathrm{N}^{\prime}-$ methylene-bis-acrylamide, $7.17 \mathrm{~g}$ of urea, $2 \mathrm{mg}$ of $\mathrm{Fe}_{2}\left(\mathrm{SO}_{4}\right)_{3} \times 9 \mathrm{H}_{2} \mathrm{O}, 80 \mathrm{mg}$ of ascorbic acid and $0.26 \mathrm{~g}$ of aluminum lactate. All the reagents were dissolved in $100 \mathrm{ml}$ of the aluminum lactate buffer. $25 \mu \mathrm{l}$ of $15 \%$ hydrogen peroxide was added to $100 \mathrm{ml}$ gel solution for polymerization. Electrophoretic cameras of VE-20 models (Helicon, Russia) with the size of the formed plate $178 \times 175 \times 1.5 \mathrm{~mm}$ were used for carrying out electrophoresis. Electrophoresis was performed at a constant voltage of $500 \mathrm{~V}$ for 3.5-4.0 hours. Fixation and staining of the gel plates was performed in $10 \%$ solution of trichloroacetic acid with the addition of $0.05 \%$ of Coomassie of brilliant blue R-250 in ethanol, for 8 hours. Identification of allelic variants of components blocks controlled by avenin-coding loci was carried out on the basis of the catalog developed by V. A. Portyanko and others [6]. Electrophoregrams of samples were recorded in the form of genetic formulas. For this purpose, the combination of $A v$ was written in a string, and then - a letter denoting the appropriate loci $(A, B$ и $C)$ and the sequence number of the allelic version of the block of the prolamins components controlled by the corresponding loci. As a standard, oats grains of Astor seed variety (Avn A2 B4 C2) were used. The computer matrix of the initial data was compiled on the basis of the obtained avenin electrophoretic spectra; the presence of the component was indicated by 1 , and the absence by 0 . Protein fractions were distinguished among themselves based on the velocity of their motion in a gel carrier. To determine the degree of genetic differentiation of samples, the data obtained by the matrix was processed by cluster analysis. The Dice coefficient was used as the similarity index, which is also known as the Nei-Li coefficient (1):

$$
S=\frac{2 n_{a b}}{n_{a}+n_{b}}
$$


where $n_{a}$ and $n_{b}$ - is the number of components present in spectra $\mathrm{A}$ and $\mathrm{B}$, respectively, and $n_{a b}$ is the number of components common to the two spectra [8]. Genetic distances $(d)$ to construct a dendrogram were calculated using the formula (2):

$$
d=1-S
$$

The method of Unweighted Pair-Group Method with Arithmetic Mean (UPGMA) [9] was used for clustering. The construction of the dendrogram was performed using the MEGA 6.06 program.

\section{Results and discussion}

As a result of the analysis it was found that 29 of the studied varieties were homogeneous in terms of the composition of avenin. From 2 to 5 biotypes were parts of heterogeneous varieties. Heterogeneity of varieties is explained by the peculiarities of breeding. For example, when creating varieties by hybridization and selection from hybrid populations, there is a high probability of selection of heterozygous plants, including the genes of prolamins. The number of biotypes that differ in the spectra of spare proteins in the resulting variety will depend on how many prolamin-coding loci was heterozygous ancestral plant. In order to more fully evaluate the degree of genetic similarity detected in all biotypes, they were considered by us as independent samples. Biotypes of one variety were numbered, starting with the most common. As a result of processing data on the component composition of the samples studied by cluster analysis, we obtained a dendrogram, presented in figure 2 .

All analyzed biotypes were United into two large clusters (Dice $=0.40)$. The exception was the second biotype of Megion, which took a stand-alone position (Dice $=0.49$ ). It should be noted that biotypes of some heterogeneous varieties were located in different groups and even clusters. This indicates a high level of genetic differences within varieties. Based on the data on the avenin composition, we have identified the following varieties with a high level of intersort genetic differences: Megion, Fobos, Local (K-8427), Uspekh, Otrada, Pushkinskij. These varieties are especially valuable for agriculture as a high level of genetic diversity is a prerequisite to increase crop yields, improve grain quality indicators and increasing the resistance of plants to adverse environmental factors $[10,11]$. It should be noted that currently the proportion of heterogeneous varieties among zoned increases, as they have an advantage over homogeneous varieties in response to various stress factors.

The clusters of obtained dendrogram consist of sub-clusters and smaller groups of samples are genetically close to each other. There are 3 groups of samples that have identical of avenin spectra. The value of the genetic distance by the Dice coefficient between them is 0 . To samples that have identical spectra are: grade Astor, Mutica 556, a third biotype of the Pushkinskij variety and second biotype of Golec variety, the genetic formula of avenin of these varieties - Avn A2 B4 C2; Persheron and Vyatskyj golozyornyj - Avn A2 B4 C1; the second biotypes of Lgovskij 9 and Pushkinskij varieties - Avn A2 B1 C3. The reasons for the identity of the spectra of spare proteins can be common origin of samples, and selection in the breeding process of genotypes with such associations of genes, which correspond to certain variants of blocks of prolamins components. 


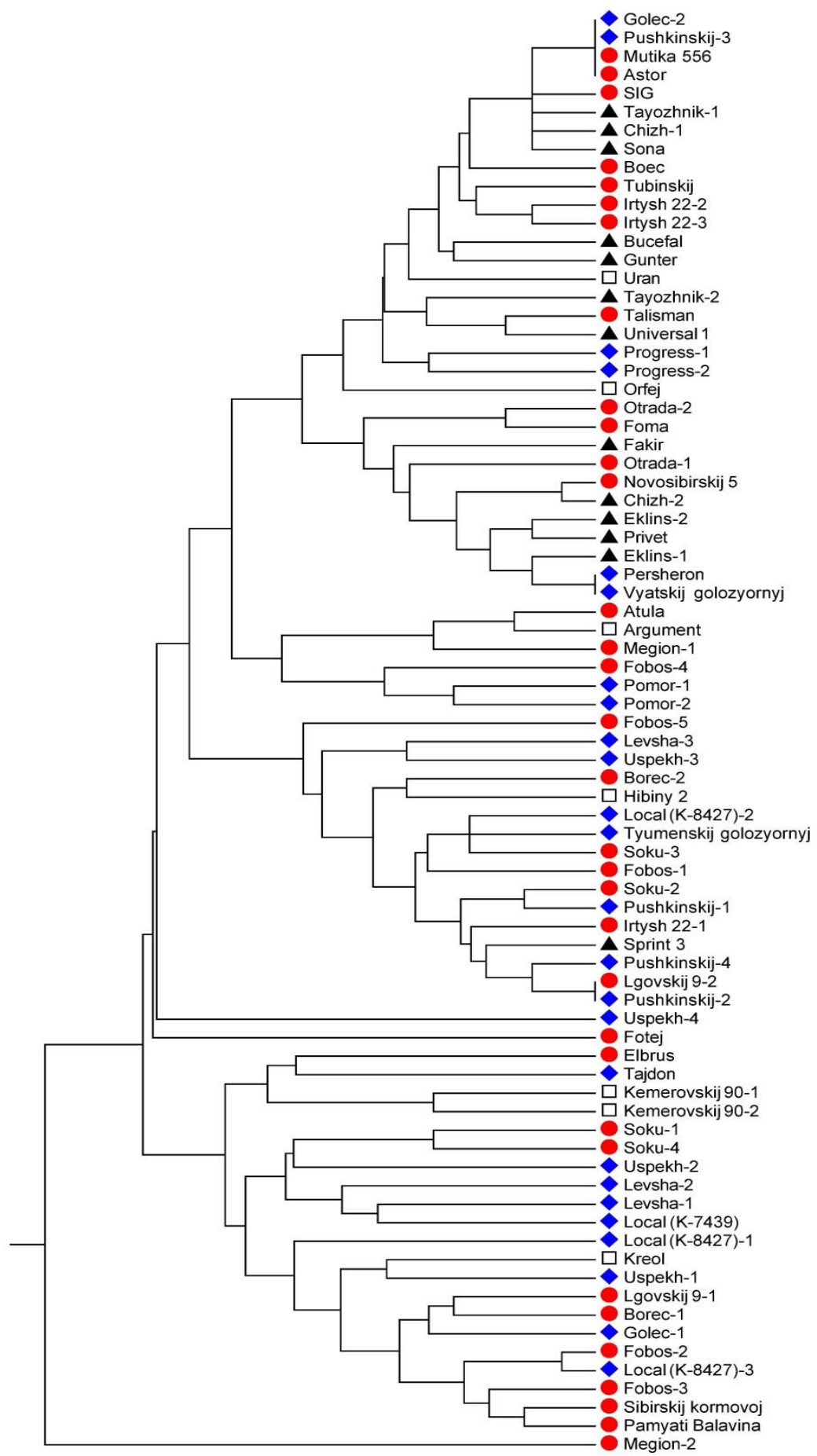

Fig. 2. Dendrogram of samples of common oats Russian selection, built on the basis of data on the component composition of avenin.

Conventions: $\boldsymbol{\Delta}$ - aurea; $\square-$ aristata; - inermis; $\bullet-$ mutica; K-xxxx - sample number in the catalog of The N.I. Vavilov All-Russian Institute of plant genetic resources (Russia). The hyphen number indicates the biotypes. The scale shows the value of the genetic distance by Dice. 
An important property of prolamins is their exposure to the action of selection, as a result of which, in certain conditions of the environment, genotypes with a non-accidental stable combination of genes are created - adaptive gene complexes [12]

At the same time, adaptive characteristics of populations correspond to common locally common marking alleles, which are the most valuable from a practical point of view. Thus, the dependence of the frequency of occurrence of different alleles of wheat, oats and barley prolamin-coding loci on agroclimatic and soil conditions was established. A. Yu. Novoselskaya-Dragovich with co-authors in the study of gliadin-coding loci of varieties of soft wheat found that based on the genetic gliadin formula and biotype composition of the sample it is possible to determine its affiliation to a particular breeding centers. At the same time, up to $5 \%$ of varieties analyzed by the authors of origin from one breeding center had identical spectra of gliadin [13]. In our opinion, the samples revealed by us with identical spectra of avenin have similar or even identical economic and valuable signs, also biological and adaptive properties. In the case of coincidence of electrophoretic prolamins spectra the differences between the varieties can be identified with the use of other genetic polymorphic marker systems, such as microsatellite (SSR) DNA markers.

As a result of the analysis of the obtained dendrogram, it was found that there is no dependence between the distribution of samples in groups and sub-clusters and their belonging to a certain species. Therefore, a method of electrophoresis of oats prolamins allows you to effectively distinguish between varieties belonging to the same species and indistinguishable by morphological characters.

\section{Conclusion}

In the modern agribusiness working in the sphere of production of high-quality seed, the method of prolamins electrophoresis is an indispensable technology for varietal identification and determination of varietal purity. This method is in great demand in the agricultural selection at creation new varieties and hybrids of grain crops. Electrophoretic analysis of oat prolamins can be successfully used in the creation of new varieties with a certain set of features and properties required for a specific natural and climatic zone. This makes this method very popular among breeding centers and other scientific organizations. As a result of the analysis of the component composition of prolamins, we have identified varieties of oats with a high level of intravarietal genetic differences: Megion, Fobos, Local (K-8427), Uspekh, Otrada, Pushkinskij. We found groups of varieties with identical component composition of spare proteins. Their genetic formula: Avn A2 B4 C2, Avn A4 B4 C2, Avn A2 $B 4 C 1$ or $A v n A 2 B 1 C 3$. As a result of the carried-out researches, it is established that the grades of oats relating to one variety and indistinguishable on morphological signs, accurately differ on component structure of avenin. This makes electrophoresis of spare proteins the most effective technology in the modern agricultural business, allowing seed enterprises to carry out laboratory varietal control at all stages of the production of seed material.

\section{References}

1. E.D. Badaeva, O.Y. Shelukhina, O.S. Dedkova, V.A. Pukhalskyi, I.G. Loskutov, Russ. J. Genet. 47, 691-702 (2011), DOI: 10.1134/S1022795411060068

2. A.Y. Novoselskaya-Dragovich, A.A. Shishkina, V.A. Melnik, V.P. Upelniek, A.V. Fisenko, L.V. Dedova, A.M. Kudryavtsev, L.A. Bespalova, Russ. J. Genet. 51, 262-271 (2015), DOI: 10.1134/S1022795415030102 
3. Y. Li, Y. Song, R. Zhou, G. Branlard, J. Jia, Plant Breed. 128(3), 235-243 (2009), DOI: $10.1111 / \mathrm{j} .1439-0523.2008 .01578 . \mathrm{X} /$

4. M. Utebayev, S. Dashkevich, A. Babkenov et al., Acta Physiologiae Plantarum. 38 (8) (2016), DOI: 10.1007/s11738-016-2209-4

5. A.A. Nikolaev, V.A. Pukhalskyi, V.P. Upelniek, Russ. J. Genet. 45(2), 189-197 (2009), DOI: 10.1134/S1022795409020094

6. V.A. Portyanko, A.A. Pomortsev, N.A. Kalashnik, V.I. Bogachkov, A.A. Sozinov, Russ. J. Genet. 23(5), 845-853 (1987) [in Russian]

7. ISO 8981 (1993) Wheat-identification of varieties by electrophoresis. http://www.iso.org/iso/catalogue_detail.htm?csnumber=16506

8. M. Nei, W. Li, Proc. Natl. Acad. Sci. USA. 76, 5269-5273. (1979).

9. P. Sneath, R. Sokal, Numerical taxonomy. The principles and practice of numerical classification (1973)

10. E.V. Metakovsky, C.W. Wrigley, F. Bekes, R. Aust. J. Agric. Res. 41(2), 289-306 (1990), DOI: 10.1071/AR9900289

11. A. Pomortsev, B. Kalabushkin, E. Lyalina, Russ. J. Genet. 37(11), 1279-1285 (2001), DOI: 10.1023/A:1012509125810

12. A.M. Kudryavtsev, L.V. Dedova, V.A. Melnik, A.A. Shishkina, V.P. Upelniek, A.Y. Novoselskaya-Dragovich, Russ. J. Genet. 50(5), 483-488 (2014), DOI: 10.1134/S1022795414050093

13. A.Y. Novoselskaya-Dragovich, A.V. Fisenko, V.A. Puhalskyi, Russ. J. Genet. 49(5), 487-496 (2013), DOI: 10.1134/S1022795413020087 